\title{
Neonatal meningococcal conjunctivitis associated with meningococcal meningitis
}

\author{
M Ellis, A M Weindling, D C Davidson, N Ho, V Damjanovic
}

\begin{abstract}
Two infants are described in whom identical strains of meningococcus were isolated from both the eyes and the cerebrospinal fluid. This suggests that the eye may be a portal of entry in at least some cases of perinatally acquired neonatal meningococcal disease and has important implications for the management of perulent conjunctivitis in the newborn.
\end{abstract}

(Arch Dis Child 1992;67:1219-20)

Around 12000 babies are born each year in the three maternity hospitals in Liverpool. Over the last five years we have encountered two babies who developed meningococcal conjunctivitis before developing meningococcal meningitis. We describe these infants here first, as a reminder to clinicians not to underestimate the potential seriousness of neonatal conjunctivitis, and second, because these cases demonstrate a possible mechanism for the development of meningococcal meningitis.

\section{Case reports}

CASE 1

This baby girl was born at term by assisted vaginal delivery to a healthy 19 year old primigravida woman after an uneventful pregnancy. The amniotic membranes were ruptured artificially 12 hours before delivery. At birth the infant was in good condition (Apgar score 9 at 1 minute) and weighed $3500 \mathrm{~g}$.

On the second day the left eye became inflamed and a swab was taken for bacteriological culture before applying neomycin eyedrops. At 60 hours of age she had a generalised seizure lasting 30 seconds and signs of meningism developed. The lumbar cerebrospinal fluid was purulent with a white cell count $3040 / \mathrm{mm}^{3}$ with numerous Gram negative diplococci. The infant was treated with cefotaxime and ampicillin for 14 days and anticonvulsant therapy for two weeks. She subsequently made an uncomplicated recovery.

The mother became unwell on the same day as her child with a fever and lower abdominal discomfort. A high vaginal swab was taken from her, and nose and throat swabs taken from the immediate family. She was first given a course of intravenous metronidazole and cefuroxime and, after identification of the organism, both parents received rifampicin.
Neisseria meningitidis group C type 2 a was cultured from the infant's eye swab, blood, and cerebrospinal fluid as well as from the mother's vaginal swab and the father's throat swab.

\section{CASE 2}

This boy was born at 37 weeks' gestation to a healthy 18 year old primigravida woman after an uneventful pregnancy. The amniotic membranes were ruptured artificially eight hours before delivery. Diminished beat-to-beat variability of the fetal heart rate developed during the first stage of labour. The infant was born in poor condition weighing $2900 \mathrm{~g}$ and required resuscitation but not ventilation. The Apgar scores were 4 at 1 minute, 5 at 5 minutes, and 8 at 10 minutes. At 12 hours of age he had a brief generalised seizure lasting 20 seconds from which he immediately recovered and remained completely well for the next two days.

On day three he developed a purulent bilateral eye discharge from which swabs were taken and neomycin eye ointment commenced. By 72 hours of age he was handling less well with poor peripheral perfusion and increasingly abnormal jerky movements. Lumbar puncture yielded cloudy cerebrospinal fluid with a white cell count of $146 / \mathrm{mm}^{3}$ with profuse Gram negative diplococci. The $\mathrm{C}$ reactive protein rose from $30 \mu \mathrm{g} / \mathrm{l}$ on day 3 to $145 \mu \mathrm{g} / \mathrm{l}$ on day 4 .

The infant received a seven day course of benzylpenicillin and cefotaxime and was treated with various anticonvulsants before the seizures came under control. After the finding of Gram negative diplococci in the infant's cerebrospinal fluid a high vaginal swab was taken from the mother, who had remained well, and throat swabs were taken from the immediate family members. They were all treated with rifampicin as prophylaxis.

$N$ meningitis group W 135 type P16 was subsequently cultured from the eye swab, blood, and cerebrospinal fluid of the infant. On this occasion the mother's vaginal and throat swabs failed to yield the organism.

\section{Discussion}

Neonatal meningococcal meningitis is rare. In 1988 Chugh et al reviewed 35 cases that had been reported since $1911,{ }^{1}$ and only three cases were found in a retrospective study of 280 cases of neonatal bacterial meningitis. ${ }^{2}$ Two cases of neonatal meningococcal meningitis associated with maternal carriage of an 
identical organism in the birth canal have previously been reported. ${ }^{34}$ In both cases the infant died. Case 1 therefore constitutes to our knowledge the third (and first reported surviving) case infected in these circumstances.

It is likely that asymptomatic carriage of the meningococcus in the genitourinary tract is increasing. A screening programme for gonorrhoea in New York in 1975 identified a rising incidence of $N$ meningitidis isolated from the genitourinary tract. ${ }^{5}$ Another North American study found the $1-2 \%$ of all urethral isolates of neisseria were non-gonoccoccal. ${ }^{4}$

There are biological similarities between Neisseria gonorrhoeae and $N$ meningitidis. Gonococcal meningitis in neonates associated with gonococcal ophthalmia neonatorum has been described, and meningococcal conjunctivitis associated with meningitis in a 7 month old infant has been reported. A 13 day old infant who died of meningococcal septicaemia two days after developing a meningococcal conjunctivitis has also been reported, but in that case the cerebrospinal fluid was sterile.

Meningococcal conjunctivitis presented on days 2 and 3 in the two cases reported here.
Within 24 hours both infants suffered a marked clinical deterioration accompanied by generalised seizures and bacteriologically confirmed meningococcal meningitis. This suggests that the eye may have been the portal of entry after intrapartum contamination with the organism.

These two cases demonstrate that the neonate is vulnerable to less pathogenic strains of $N$ meningitidis which can be found in the birth canal. Swabs from purulent neonatal conjunctivitis should be immediately examined by Gram staining. If Gram negative diplococci are demonstrated, parenteral antibiotic treatment is advisable until culture identification is available.

1 Chugh K, Bhalla CK, Joshi KK. Meningococcal brain abscess and meningitis in a neonate. Pediatr Infect Dis 1988;7:136-7.

2 Mulder CJJ, Zanen HC. A study of 280 cases of neonatal meningitis in the Netherlands. $\mathcal{F}$ Infect 1984;9:177-84.

3 Sunderland WA, Harris HH, Spence DA, Lawson HW. Meiningococcaemia in a newborn infant whose mother had meningococcal meningitis. F Pediatr 1972;81:856.

4 Jones RN, Slepack J, Eades A. Fatal neonatal meningococcal meningitis. $\mathscr{f} A M A$ 1976;236:2652-3.

5 Faur YC, Weisburd MH, Wilson ME. Isolation of neisseria meningitidis from the genitourinary tract and anal canal. f Clin Microbiol 1975;2:178-82.

6 Holmberg L, Moestrup T. Meningitis following conjunctivitis. $\mathcal{F}$ Pediatr 1974;94:339. 